\title{
Development and application of a strategic modelling tool for the evaluation of air pollution mitigation strategies for urban freight in Sydney
}

\section{S. Greaves}

The Institute of Transport \& Logistics Studies, Faculty of Economics \& Business, University of Sydney, Australia

\begin{abstract}
This paper details the development of a strategic-level modelling tool designed to provide a flexible approach for decision-makers to evaluate the air quality and greenhouse gas mitigation (GHG) impacts of mitigation strategies for urban freight. The model, known as STEAR-F (Strategic Tool for the Environmental Analysis of Road Freight) combines available information on freight travel, fleet characteristics, and emission factors to provide estimates of GHG and non-GHG emissions. Following details of the model development, STEAR-F is applied to evaluate strategies currently under consideration in Sydney. These include accelerated vehicle scrappage programs, diesel retrofit programs, Low Emission Zones (LEZs), alternative fuel programs, and educational/information programs. Results show that by 2015, while net reductions in non-GHG emissions are anticipated as the fleet modernises and new emissions standards come into force, GHG emissions are expected to grow by $40 \%$ due to the increase in commercial vehicle kilometres travelled (CVKT). Scenario testing suggests that accelerated scrappage programs, retrofit programs, and LEZs, could achieve further reductions in non-GHGs of 5-10\% respectfully. Alternative fuel programs are anticipated to reduce some of the reliance on fossil fuels, but emissions benefits are marginal due to lower operational efficiencies and fuel life-cycle concerns. A package of educational/informational strategies could achieve savings of around $10 \%$ in GHGs assuming relatively conservative rates of participation.
\end{abstract}

Keywords: greenhouse gases; air quality; road freight. 


\section{Introduction}

As with most major metropolitan areas around the world, Sydney has witnessed a rapid growth in urban freight movements and commercial vehicle kilometres of travel (CVKT). Between 1990 and 2003, the volume of freight moved by road in Sydney increased by 59\%, while CVKT increased by 37\% (Bureau of Transport and Regional Economics - BTRE, 2006). This growth in commercial vehicle activity has exacerbated concerns over greenhouse gas (GHG) and air quality impacts - for instance, in 2006 freight vehicles were deemed responsible for $33 \%$ of GHGs, $39 \%$ of Nitrous Oxides (NOx) and $65 \%$ of particulate matter (PM) coming from the transport sector in Sydney (New South Wales Department of Environment and Climate Change - NSW DECC, 2007). Looking ahead, CVKT is projected to grow by around $35-40 \%$ by 2015 , which threatens to more than offset anticipated gains from the adoption of new emissions and fuelefficiency standards for freight vehicles.

Various technological, educational, regulatory and market-based strategies have been proposed to tackle such problems (Vieira et al. 2007). However, while evidence of the effectiveness of these strategies from an air quality perspective continues to grow (Anderson et al. 2005), few analytical tools exist that provide policy-makers with the means to systematically quantify their emissions benefits. The current paper describes the development and application of a strategic-level modelling tool designed to assist policy-makers evaluate the air quality and greenhouse gas impacts of urban freight mitigation strategies. The approach, termed STEAR-F (Strategic Tool for the Environmental Analysis of Road Freight) combines commercial vehicle traffic estimates with information on fleet characteristics (vehicle type, age, fuel type, scrappage/replacement) and emission factors to provide estimates of Greenhouse Gas (GHG) and non-GHG emissions. Operating as a Visual Basic Application (VBA) within a spreadsheet environment, STEAR-F is designed to provide a quick response to what-if questions about mitigation strategies. Summaries of commercial vehicle kilometres travelled (CVKT) and emissions can be reported out at various spatial levels including Local Government Area (LGA), Statistical Division (SD) and the Sydney Greater Metropolitan Area (GMA) (The Sydney GMA comprises the Statistical Divisions (SDs) of Sydney, Hunter and Illawarra.).

This paper is organised as follows. First, more details are provided on the methodological development of STEAR-F. Following this the model is used to evaluate example strategies currently under consideration in Sydney including a) accelerated vehicle scrappage programs, b) an expansion of existing diesel vehicle retrofit programs, c) a Low Emission Zone around the central area of the city, d) expansion of alternative fuel programs, and e) development of educational/information programs focused on driver behaviour, new technologies and proactive vehicle maintenance. Finally, some concluding remarks about future developments of the approach are provided. 


\section{Model development}

The major components of STEAR-F are shown in Figure 1 together with the required data, which is all publicly available. Each of these components is now briefly described.

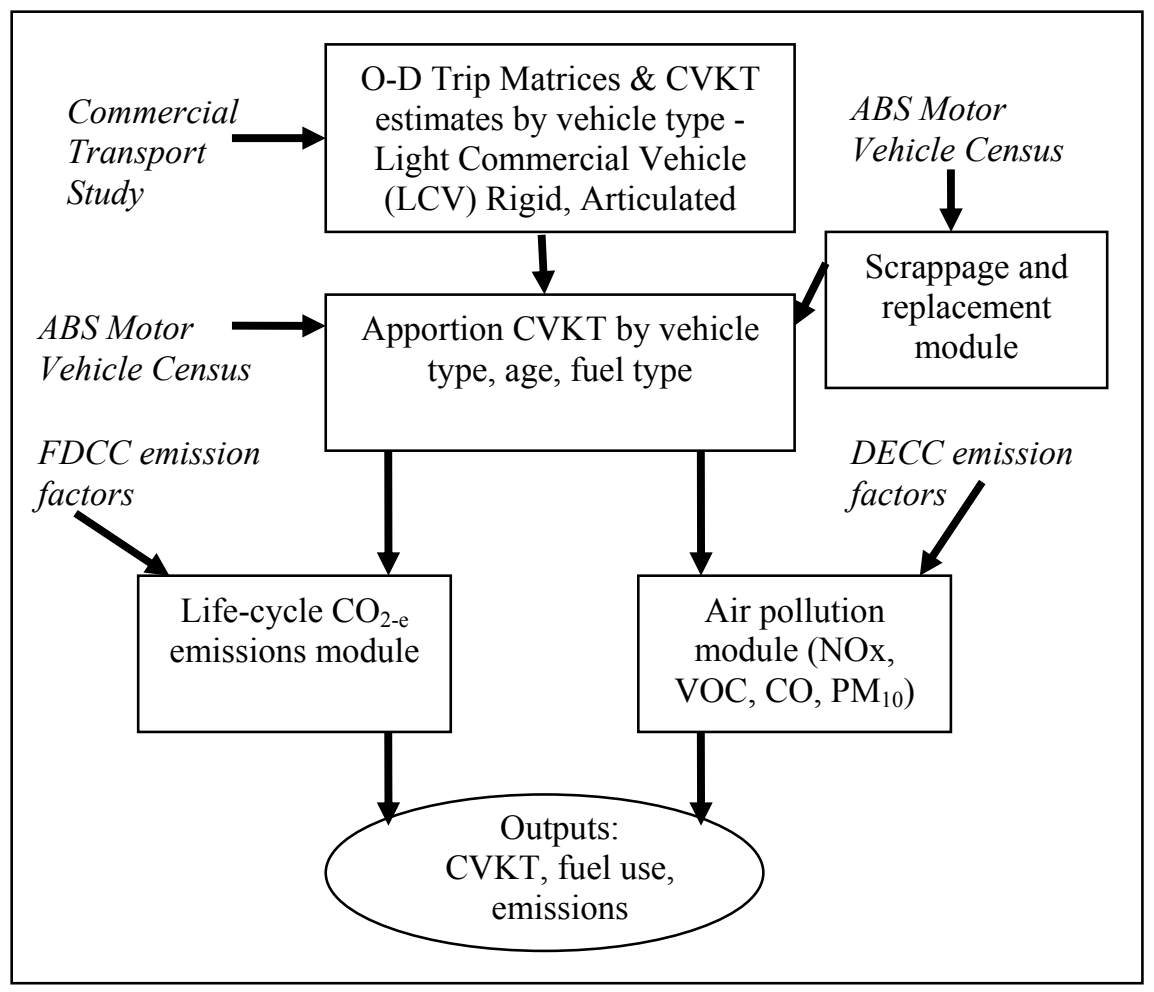

Figure 1: Overview of the STEAR-F post-processor modelling approach.

\subsection{Origin-destination (O-D) trip and CVKT matrices}

O-D matrices by each of the three vehicle types were provided by the New South Wales Transport Data Centre (TDC), the agency responsible for preparation of such forecasts in Sydney. The TDC estimates are prepared using a four-step fre ;ht model, which has been progressively refined since the mid-1990s with be...r data on commodity movements (Mendigorin and Peachman, 2005). The trip tables provide average weekday movements between the 1,129 traffic zones covering the Sydney GMA for three vehicle types, namely Light Commercial Vehicles (LCVs), rigid trucks, and articulated trucks.

\subsection{Apportionment of CVKT by vehicle type, age and fuel type}

In addition to vehicle type, vehicle emissions vary significantly by the number of kilometres driven and fuel type used. It was therefore necessary to apportion the 
CVKT computed in the previous step for each of the three vehicle types by vehicle age (proxy for number of kilometres driven) and fuel type. As this information was not directly known, a process was employed to indirectly apportion the CVKT based on information on CVKT derived from the Australian Bureau of Statistics (ABS) Survey of Motor Vehicle Use - SMVU (ABS, 2007a). The SMVU provides CVKT estimates based on a quarterly sample of selfreported vehicle odometer readings from around Australia. Unfortunately, it does not include fuel type and is subject to high standard errors resulting from very small sample sizes when the data are used at a disaggregate level. Consequently, these data were supplemented using information from the ABS Motor Vehicle Census (MVC), which provides a full enumeration of registered vehicles down to postcode level by vehicle type, age, and fuel type (ABS, 2007b).

\subsection{Life-Cycle $\mathrm{CO}_{2}$-e emissions module}

The computation of $\mathrm{CO}_{2}$-e emissions involved a two-step process. The first step was to establish the fuel efficiency by vehicle type and age using information from the BTRE, the primary source of such data in Australia (BTRE, 2006). These figures were then multiplied by the latest $\mathrm{CO}_{2}$-e estimates provided by the Federal Department of Climate Change (FDCC, 2008), to derive a $\mathrm{CO}_{2}$-e g/km for each vehicle type and year of manufacture.

\subsection{Air pollution module}

Estimates of NOx, VOCs, $\mathrm{CO}$, and $\mathrm{PM}_{10}$ emissions for petrol and diesel were derived using emission factors provided by the NSW DECC. The factors are based on local emissions test data supplemented by evidence from the US and Europe. For alternative fuels, the only source of Australian information is that provided by a large study of heavy vehicles at the turn of the millennium (Beer et al. 2000). It must be noted these factors represent fleet averages, with no breakdown by vehicle age and type so clearly must be used with caution.

\subsection{Vehicle scrappage and replacement module}

This module is designed to capture the turnover that occurs as older vehicles are retired and newer vehicles enter the fleet. The previously noted ABS Motor Vehicle Census provides annual registered vehicle type/age breakdowns up to 2007 from which empirically-derived scrappage rates were obtained. Future year (i.e., beyond 2007) scrappage rates were determined by trend-line extrapolation from the existing data.

\section{Results}

\subsection{Base case}

The 2003 Base Case provided a starting point for the analysis - a summary of results is shown in Table 1. Some points are worthy of note. First, LCVs are 
responsible for more than three-quarters of trips and over half the CVKT in the Sydney GMA, a reflection of the demand for quicker, more frequent deliveries of smaller loads. Second, rigid and articulated trucks are responsible for the majority of $\mathrm{NOx}(85 \%)$ and $\mathrm{PM}_{10}(72 \%)$, while LCVs are responsible for the majority of $\mathrm{CO}(63 \%)$. This primarily reflects the predominantly diesel-based heavy-duty vehicle fleet and still largely petrol-driven LCV fleet (approximately two-thirds of LCVs run on petrol). Third, heavy-duty vehicles are responsible for a disproportionately high $(75 \%)$ proportion of GHGs relative to their CVKT reflective of their substantially higher fuel intensities on a per kilometer basis. This should be qualified, because (arguably) tonne-kms should be the measure used not simple kilometers. If this is the case, heavy-duty vehicles work out be around 5-10 times more efficient in terms of GHGs/tonne-km.

Table 1: 2003 Base Case Estimates in the Sydney GMA.

\begin{tabular}{|l|l|l|l|l|}
\hline & LCV & Rigid & Articulated & Total \\
\hline Trips & $\begin{array}{l}935,832 \\
(78 \%)\end{array}$ & $179,400(15 \%)$ & $84,479(7 \%)$ & $1,199,713$ \\
\hline CVKT & $\begin{array}{l}10,231,493 \\
(53 \%)\end{array}$ & $\begin{array}{l}5,106,992 \\
(27 \%)\end{array}$ & $\begin{array}{l}3,805,485 \\
(20 \%)\end{array}$ & $19,143,970$ \\
\hline NOx (t) & $14.4(16 \%)$ & $29.8(32 \%)$ & $48.0(52 \%)$ & 92.7 \\
\hline VOC (t) & $9.7(44 \%)$ & $8.7(40 \%)$ & $3.5(16 \%)$ & 22.7 \\
\hline PM $_{10}(\mathrm{t})$ & $101.6(63 \%)$ & $42.2(26 \%)$ & $18.0(11 \%)$ & 162.7 \\
\hline $\mathrm{CO}_{2}$-e (t) & $1.2(27 \%)$ & $1.7(39 \%)$ & $1.4(33 \%)$ & 5.0 \\
\hline CO2-e (t) & $3,202(25 \%)$ & $3,781(29 \%)$ & $5,879(46 \%)$ & 12,862 \\
\hline $\begin{array}{l}\text { Petrol } \\
(\text { litres } \\
\text { '000) }\end{array}$ & $905(73 \%)$ & $259(21 \%)$ & $75(6 \%)$ & 1,240 \\
\hline $\begin{array}{l}\text { Diesel } \\
(\text { litres } \\
\text { '000) }\end{array}$ & $277(8 \%)$ & $1,055(32 \%)$ & $1,938(59 \%)$ & 3,271 \\
\hline $\begin{array}{l}\text { Alt. Fuels } \\
\text { (litres } \\
\text { '000) }\end{array}$ & $93(50 \%)$ & $499(26 \%)$ & $45(24 \%)$ & 188 \\
\hline
\end{tabular}

*Results for an average weekday; Alt. fuels include LPG and biodiesel only.

\subsection{Business as usual (2015BAU)}

The 2015 Business as Usual (BAU) strategy formed the baseline for evaluating the various strategies. Results are shown in Table 2. Clearly, large increases in trips and CVKT, particularly within the LCV and articulated truck fleet are predicted. Commensurate increases are predicted for GHGs, because (in effect) none of the BAU emissions standards target GHGs and in fact new emissions standards impose small fuel and GHG penalties. Current trends towards increasing diesel fuel use are predicted to continue although a significant 
proportion of the LCV fleet is predicted to remain petrol-driven. A final point of note is that even with the large increases in CVKT, the tightening of Australian emissions standards for new vehicles in line with Euro IV and eventually Euro V are anticipated to lead to significant net reductions in the 'problem' diesel pollutants of $\mathrm{NOx}$ and $\mathrm{PM}_{10}$.

Table 2: $\quad 2015$ Business as usual estimates in Sydney GMA.

\begin{tabular}{|c|c|c|c|c|}
\hline & LCV & Rigid & Articulated & Total \\
\hline Trips & $\begin{array}{l}1,354,846 \\
(80 \%)\end{array}$ & $200,666(12 \%)$ & $134,370(8 \%)$ & $1,689,882$ \\
\hline CVKT & $\begin{array}{l}14,910,871 \\
(56 \%)\end{array}$ & $\begin{array}{l}5,638,064 \\
(21 \%)\end{array}$ & $\begin{array}{l}5,907,105 \\
(22 \%)\end{array}$ & $26,456,042$ \\
\hline $\mathrm{NOx}(\mathrm{t})$ & $16.9(23 \%)$ & $21.8(30 \%)$ & $34.0(47 \%)$ & 73.2 \\
\hline $\operatorname{VOC}(\mathrm{t})$ & $11.4(63 \%)$ & $3.8(21 \%)$ & $2.9(16 \%)$ & 19.0 \\
\hline $\mathrm{CO}(\mathrm{t})$ & $175.1(88 \%)$ & $10.8(5 \%)$ & $12.8(6 \%)$ & 199.6 \\
\hline $\mathrm{PM}_{10}(\mathrm{t})$ & $1.3(53 \%)$ & $0.7(26 \%)$ & $0.5(22 \%)$ & 3.3 \\
\hline $\mathrm{CO}_{2}-\mathrm{e}(\mathrm{t})$ & $4,566(25 \%)$ & $4,084(23 \%)$ & $9,282(52 \%)$ & 17,932 \\
\hline $\begin{array}{l}\text { Petrol } \\
\text { (litres } \\
\text { '000) } \\
\end{array}$ & $1,116(91 \%)$ & $47(4 \%)$ & $59(5 \%)$ & 1,224 \\
\hline $\begin{array}{l}\text { Diesel } \\
\text { (litres } \\
' 000) \\
\end{array}$ & $540(11 \%)$ & $1,342(27 \%)$ & $3,108(62 \%)$ & 4,991 \\
\hline $\begin{array}{l}\text { Alt. Fuels } \\
\text { (litres } \\
\text { '000) }\end{array}$ & $140(53 \%)$ & $46(17 \%)$ & $77(29 \%)$ & 263 \\
\hline
\end{tabular}

*Results for an average weekday; Alt. fuels include LPG and biodiesel only.

\subsection{Scenario testing}

Various scenarios were formulated and tested using STEAR-F including a) an accelerated scrappage program, b) an expansion of existing diesel vehicle retrofit programs, c) a Low Emission Zone around the central area of Sydney, d) expansion of alternative fuel programs, and e) development of educational/information programs focused on driver behaviour, new technologies and proactive vehicle maintenance. Each of these is now described before a summary of results is presented.

Accelerated scrappage programs are designed to encourage the retirement of older, high-emitting vehicles and (potentially) their replacement with newer vehicles through a variety of fiscal mechanisms. Currently, no such programs exist in Sydney although it has been discussed on numerous occasions (e.g., BTRE, 2006). STEAR-F was used to assess the impacts of doubling the natural vehicle scrappage rates of pre-Euro III standard vehicles with varying rates of replacement with new vehicles. 


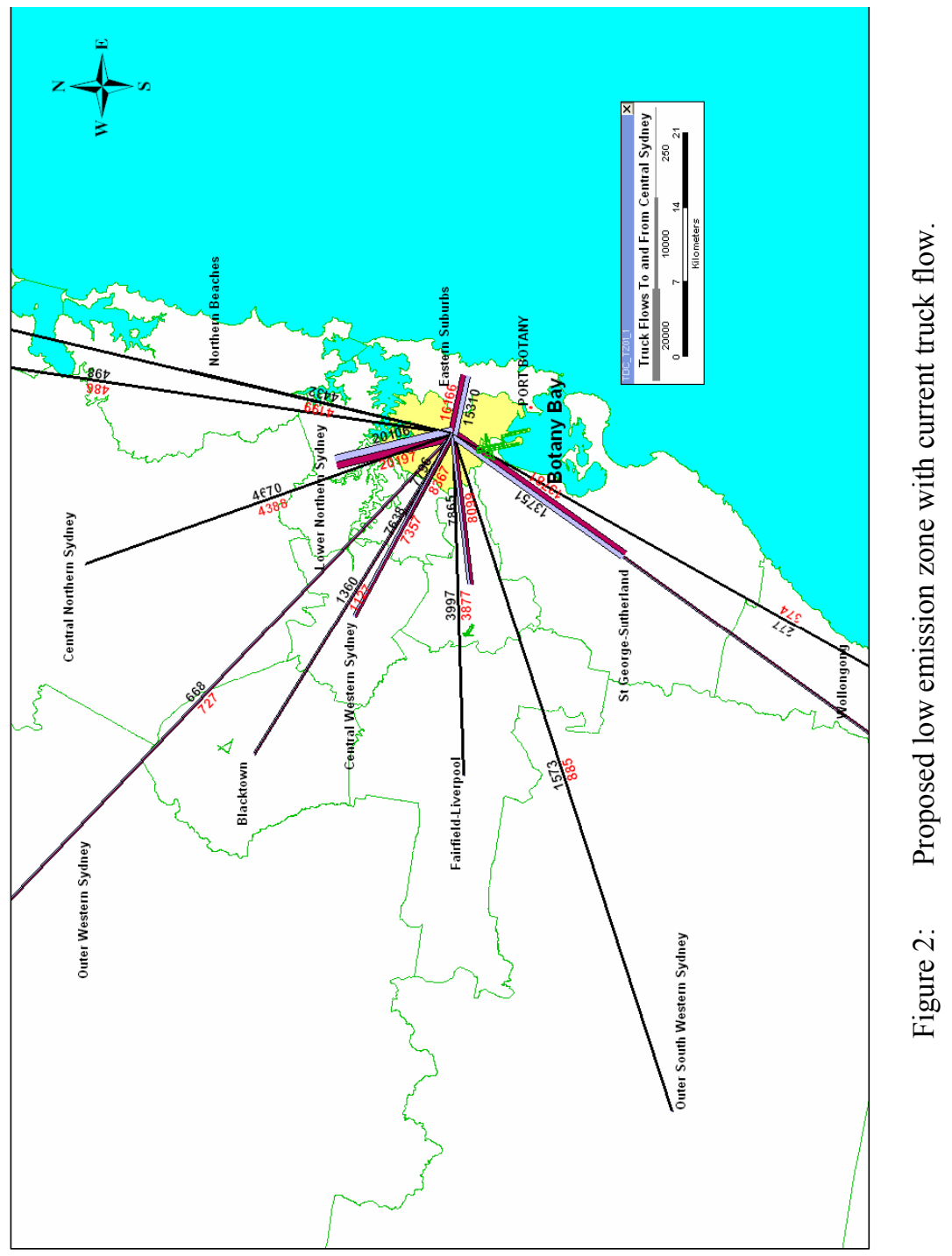


The expanded diesel retrofit program again targeted pre-Euro III vehicles, but this time considered the impacts of substantially increasing the rates of installation of partial particle traps - the New South Wales government has piloted such a program with 200 vehicles so far, which has reports partial particle traps reduce $\mathrm{PM}_{10}$ by $50 \%, \mathrm{CO}$ by $45 \%$, and impose a $1 \%$ fuel penalty, so these were the assumed savings used in STEAR-F (NSW DECC, 2007).

Low Emission Zones (LEZs) have recently been implemented in London, Berlin and Milan following earlier examples in Sweden as a mechanism for improving local air quality by targeting older, high-emitting vehicles. To demonstrate the procedure, an LEZ was introduced in 2010, targeting commercial vehicles pre-dating Euro III standards (i.e., the same criteria as the London LEZ) entering the central Sydney area (area highlighted in Figure 2).

Alternative fuel programs are currently being heavily pushed by the federal and state governments in Australia as both a mechanism to reduce dependence on foreign oil and to realize emissions benefits. Two alternative fuel scenarios were analyzed using STEAR-F. The first involved a doubling of LPG conversions within the LCV fleet as higher fuel prices and government incentives for alternative fuels take effect. The second involved increasing the use of biodiesel significantly for heavy diesel vehicles from the current $2 \%$ assumed in the 2015BAU to $20 \%$. While there is still heavy debate about biodiesel it is making small but significant inroads into the market in Australia mainly sold as B20 and B100 to niche operations such as garbage trucks.

\subsection{Scenario testing results}

Summaries of the air quality and GHG emissions of each scenario compared to the Business-as-Usual are shown in Figure 3. In each case, it is assumed the programs start in 2010 with assessments made in 2015.

The results show that under the accelerated scrappage program, reductions of $5-8 \%$ in the major pollutants are predicted, reflecting the fact that (in effect) there has been a shift in CVKT from pre- to post-Euro III vehicles. Further reductions are observed if these vehicles are replaced with new vehicles (arguably unrealistic, but never-the-less informative). The impacts of the expanded diesel retrofit strategy, suggests around a 5-7\% reduction in $\mathrm{PM}_{10}$ depending on the rate of participation.

The LEZ scenario suggests around a 3-5\% decrease in the major pollutants if all pre-Euro III vehicles are banned reflecting the tightening emissions standards from Euro III onwards. The NOx and $\mathrm{PM}_{10}$ reductions are primarily driven by the tightening of diesel standards affecting heavy trucks while the VOC and CO reductions are primarily due to tightening petrol standards affecting LCVs. The relatively small reductions in fuel consumption and GHGs are reflective of better fuel efficiency in newer model vehicles tempered by the fuel penalty associated with the retrofit technology. If the results are analysed at the LEZ level, $\mathrm{PM}_{10}$ levels are $9.4 \%$ lower than the BAU strategy, with CO (16.3\%), VOC (15.9\%), and NOx (11.5\%) respectively. The explanation behind the disproportionately 


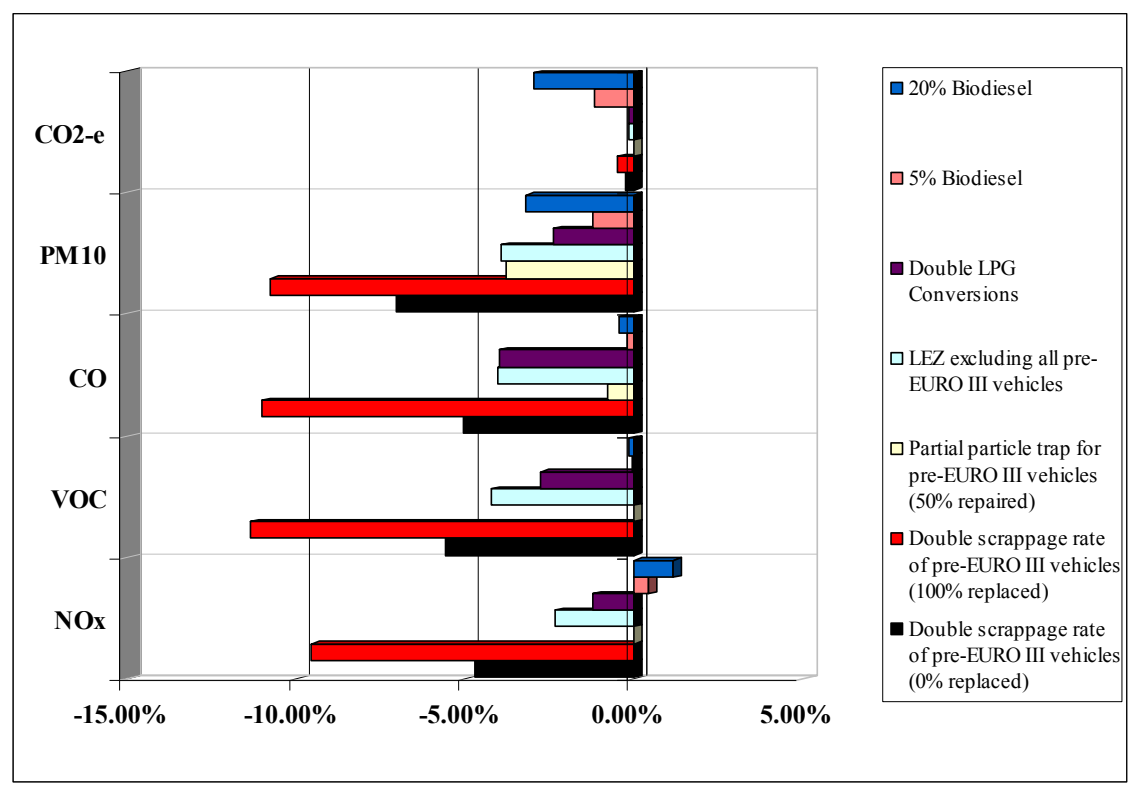

Figure 3: Air Quality and GHG emissions for each strategy compared to Business-as-Usual (2015).

large percentage reductions in VOCs and CO are because LCVs make up a greater proportion of the LEZ CVKT than the regional CVKT.

In terms of the alternative fuel strategies, a doubling of LPG vehicles results in small but notable reductions in fuel use and all the pollutants - however, the drop in GHGs is minimal. The reason for this relates to the fact that while emission factors are lower for LPG on a $\mathrm{g} / \mathrm{km}$ basis, it is around $20 \%$ less efficient than petrol and even more so for diesel. For the biodiesel scenarios (as anticipated) the main benefits are from savings of diesel fossil fuels - the reason it does not equate to a commensurate savings is because biodiesel is less efficient than diesel (although by no means to the extent of LPG to petrol). GHGs are reduced by $1-7 \%$ depending on the assumed proportions of biodiesel with reductions in all the air pollutants except NOx.

Educational and informational programs focusing on encouraging more environmentally-aware vehicle purchasing, usage, and maintenance practices are receiving increasing interest in Australia as a (relatively) low-cost approach for improving fuel efficiency and reducing GHGs in particular. STEAR-F was used to assess a number of programs, currently under consideration in Sydney including an environmentally-friendly driving program, an anti-idling campaign, the uptake of intelligent communication technologies, use of fuel-efficient tyres, and proactive maintenance practices.

The effectiveness of these types of strategies is clearly dependent on assumptions about the fuel savings and levels of participation/uptake. Given the lack of Australian information, the assumed fuel savings are based on 
assumptions from the UK Freight Best Practice Program, a widely acclaimed national repository of case studies, reports, software and multi-material applications, designed around saving fuel, and improving operational efficiency (http://freightpractice.org.uk). For the purposes of this evaluation, three levels of participation were evaluated (low, medium, high) for a package of driver training, intelligent technology programs, and tyre maintenance programs. The rationale for the participation rates was as follows:

The low participation rate scenario assumes that increasing fuel prices and growing awareness of environmental issues will lead to more (primarily) larger and medium-sized operations adopting proactive fuel maintenance strategies. Information on fuel-saving measures will increase (primarily through web-based resources), but change will be primarily voluntary.

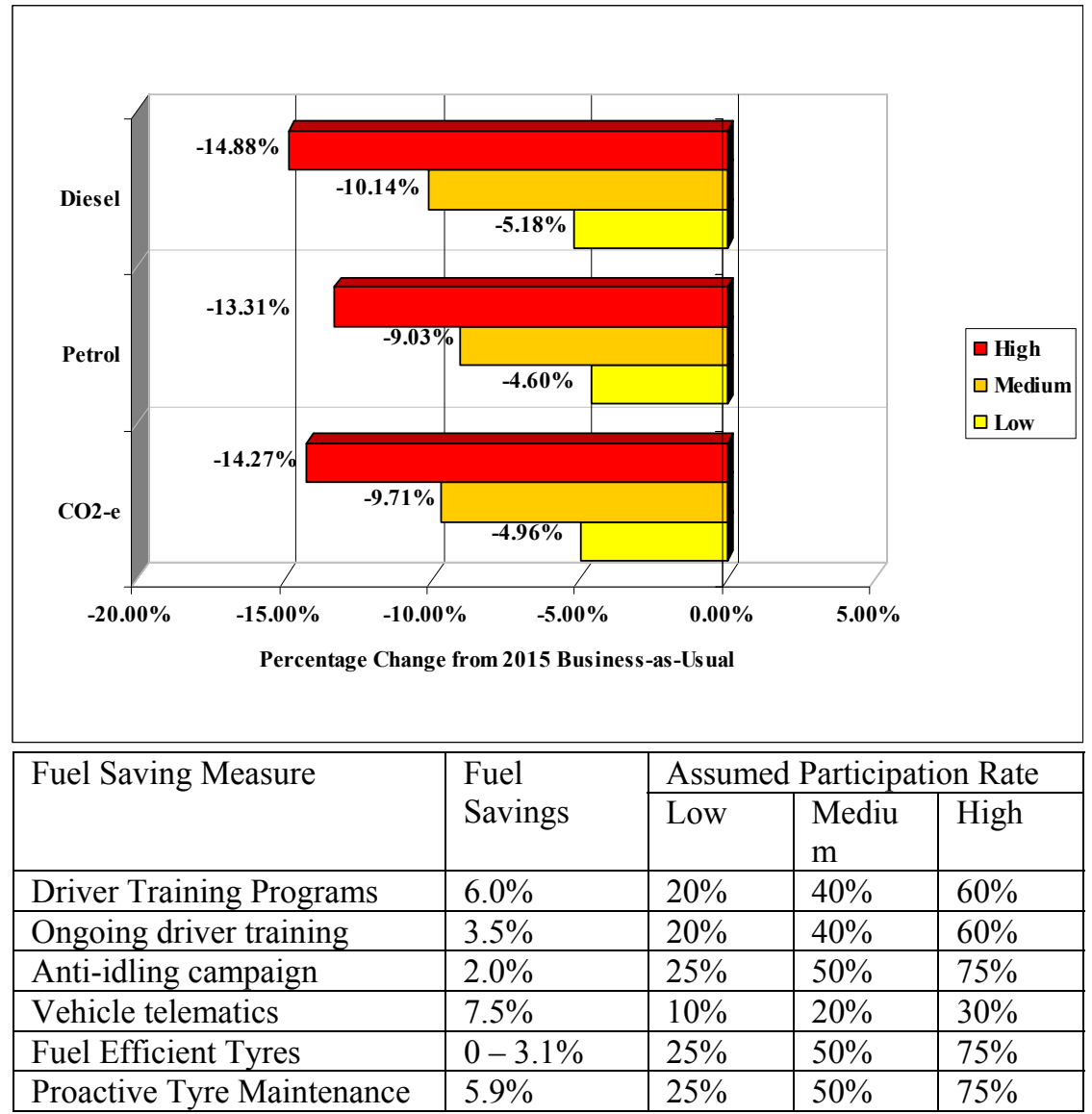

Figure 4: $\quad$ Estimated impacts educational and informational strategies.

The medium participation rate scenario assumes that formal environmentally-friendly driver training programs begin in 2010 but 
participation is voluntary. A region-wide anti-idling campaign is anticipated to begin in 2010 as awareness of the costs in terms of wasted fuel as well as emissions becomes more widely publicized. Uptake of intelligent technologies is anticipated to grow off a low base as the technology becomes more mainstream and awareness of the benefits of such systems grows. However, the cost of such systems is anticipated to remain a major deterrent for many users, restricting it to the larger operators. Developments in and adoption of fuel-efficient tyres is anticipated to grow significantly along with proactive tyre maintenance as the fuel savings become more transparent to operators.

The high participation rate scenario assumes mandating along with significant funding for environmentally-friendly driver training programs for commercial vehicle operators. The anti-idling campaign is now strictly enforced with on-the-spot fines for violators. The uptake of intelligent technologies is assisted by a rapid decrease in the costs as more suppliers enter the marketplace - however, overall utilization rates remain low because benefits appear marginal for many (small) operators. Fuel-efficient tyres and proactive tyre maintenance programs become mandatory.

The assumed participation rates for each scenario are shown in Figure 4 along with the anticipated savings in GHGs and fuel use. Accepting the caveats about the assumptions, the message is that significant savings are possible from strategies that require some behavioural modifications but do not fundamentally change the way freight transport operates and are likely to result in net cost reductions.

\section{Conclusions}

This paper details the development of a strategic-level modelling tool to evaluate the impacts of air quality and greenhouse gas mitigation strategies for urban freight. Developed from freely available information on freight travel, fleet characteristics, and emission factors, the model provides a flexible approach for decision-makers to evaluate a number of different strategies. Among the main findings of the evaluation are that under a 2015 Business-as-Usual scenario, net declines in $\mathrm{NOx}$ and $\mathrm{PM}_{10}$ are anticipated (even allowing for substantial increases in CVKT) as vehicle and fuel standards continue to tighten. However, GHGs are projected to increase dramatically because the marginal gains in new vehicle fuel efficiency will be overwhelmed by the growth in CVKT and the continued reliance on fossil fuels.

Scenario analysis using STEAR-F suggests that programs to expedite the scrappage and/or retrofit of older vehicles with particle traps could realize further savings across the Sydney GMA in the major pollutants of up to $8 \%$ depending on participation levels. A Low Emission Zone (LEZ) around the city center and port areas targeting pre-Euro III vehicles was found to realize a $10-15 \%$ reduction in major pollutants for the LEZ area. Although politically problematic, other cities such as London have demonstrated LEZs are a feasible mechanism for trying to deal with local air quality issues. Both the alternative fuel strategies considered here realized positive benefits in terms of fuel savings. However, the 
emissions benefits were lessened because these fuels are less efficient (particularly LPG) than fossil fuels on a $\mathrm{g} / \mathrm{km}$ basis. Finally, a package of educational/informational strategies were assessed and found to provide savings of around $10 \%$ in GHGs based on relatively conservative rates of participation.

\section{References}

[1] Anderson, S., J. Allen and M. Browne (2005). Urban Logistics - How Can it Meet Policy Makers' Sustainability Objectives? Journal of Transport Geography 13, 71-81.

[2] Australian Bureau of Statistics (2007a). Survey of Motor Vehicle Use. Retrieved 04/05/08 from: http:/www.abs.gov.au/AUSSTATS/abs@.nsf/ DetailsPage/9208.012\%20months\%20ended\%2031\%20Oct\%202007?

[3] Australian Bureau of Statistics (2007b). Motor Vehicle Census. Retrieved 04/05/08 from: http:/www.abs.gov.au/AUSSTATS/abs@.nsf/DetailsPage/ 9309.031\%20Mar\%202007?

[4] Australian Government Federal Department of Climate Change (2008) National Greenhouse Accounts Factors. Retrieved 06/08/08 from www.climatechange.gov.au.

[5] Beer, T, T Grant, G Morgan, L Lapszewicz, P Anyon, J Edwards, P Nelson, H Watson \& D Williams (2000). Comparison of transport fuels, final report (EV45A/2/F3C) on the stage 2 study of life-cycle emissions analysis of alternative fuels for heavy vehicles, DEWR, Canberra. Retrieved 04/05/08 from: http://www.greenhouse.gov.au/transport/comparison/index.html.

[6] BTRE (2006). Freight Measurement and Modelling in Australia. Report 112. Canberra. Retrieved 12/02/08 from: http://www.btre.gov.au/docs /reports/r112/R112.pdf.

[7] Mendigorin, L. and J. Peachman (2005) Estimation of Small-Area Commercial Vehicle Movements in the Sydney Greater Metropolitan Area: Development, Estimation Issues Addressed and Enhancements to the Estimation Method. CD-ROM Procs. of the $28^{\text {th }}$ Australasian Transport Research Forum (ATRF). Sydney.

[8] New South Wales Department of Environment and Climate Change (2007). Air Emissions Inventory for the Greater Metropolitan Region in New South Wales: On-Road Mobile Emissions Module: Results, 2007.

[9] Vieira, J. F. Moura, J.M. Viegas (2007) Transport policy and environmental impacts: the importance of multi-instrumentality in policy integration. Transport Policy 14, 421-432. 